\section{Vollzug des PFK* im Jahre 2000: Jahresbericht des Sekretariates}

\author{
F. Schwarzenbach
}

Der Pharma-Fachwerbungs-Kodex (PFK) ist in der geltenden Fassung seit fünf Jahren in Kraft (gleich lang wie die Richtlinien der IKS über die Heilmittelwerbung). Die meisten Arzneimittelhersteller und -importeure haben sich durch Unterzeichnung verpflichtet, die Regeln des PFK einzuhalten. Die Anforderungen an das Marktverhalten der Firmen werden vom Sekretariat des PFK überwacht. Es veranlasst die verantwortlichen Firmen, Kodexverletzungen zu beheben.

Im Mai dieses Jahres ist mein Vorgänger, Herr Dr. W. Forster, nach 11jähriger, engagierter Tätigkeit als Sekretär des PFK zurückgetreten. Nach einer kurzen Einarbeitungszeit habe ich diese interessante Herausforderung übernommen und hoffe, dass es mir gelingt, die wichtige Aufgabe ebenso engagiert und souverän weiterzuführen.

Im Jahre 2000 behandelte der PFK-Sekretär im Zusammenhang mit dem PFK insgesamt 171 Meldungen (im Vorjahr: 176). 92 bzw. 54\% (87 bzw. 49\%) stammten von Konkurrenten, in 64 Fällen bzw. 37\% (61 bzw. 35\%) beanstandete das Sekretariat von sich aus Promotionsmittel (Inserate, Aussendungen usw.). Die übrigen 15 (9\%) wurden von Ärzten, der IKS und weiteren Stellen eingereicht.

Nun zu den einzelnen PFK-Erfordernissen und $\mathrm{zu}$ den Verstössen dagegen: Beunruhigend ist der Anstieg von 11 im Vorjahr auf 18 Differenzen zur Arzneimittelfachinformation, wie sie von der IKS bei der Registrierung genehmigt wurde. In den meisten Fällen wurde versucht, noch nicht registrierte Indi-

* Kodex vom 1. August 1991, revidiert am 8. Juni 1995, für die Promotion und Werbung für Arzneimittel der Humanmedizin gegenüber Medizinalpersonen (Pharma-Fachwerbungs-Kodex, PFK).

Korrespondenz:

Dr. med. Felix Schwarzenbach

Schweizerische Gesellschaft für Chemische Industrie SGCI

Nordstrasse 15

Postfach

CH-8035 Zürich kationen zu bewerben. Wie 1999 wurde in 3 Fällen versucht, für ein noch nicht zugelassenes Arzneimittel Werbung zu machen. Um hier wieder mehr Klarheit zu schaffen, erstellte der Sekretär eine allgemeine Information mit Anwendungsbeispielen dazu.

In 24 Fällen (im Vorjahr in 30) waren nicht alle vom PFK geforderten Angaben vorhanden. Am häufigsten fehlte die gekürzte Fachinformation bei Mailings.

Nach dem erfreulichen Rückgang im Vorjahr gab es wieder einen leichten Anstieg von 45 auf 53 Meldungen zu den allgemeinen qualitativen Anforderungen. Unverändert 9 Promotionen mit unvollständigen Literaturangaben mussten vom Sekretär bearbeitet werden. Die Erkenntnis scheint sich mit einer einzigen Ausnahme durchgesetzt zu haben, dass wirksame Medikamente grundsätzlich unerwünschte Wirkungen haben können. Der Ausdruck «sicher» wurde nur in 2 Fällen ohne sachgerechte Qualifikation verwendet und nur in einem Fall wurde versucht anzugeben, dass ein Medikament keine Gewöhnung erzeuge. Ebenso scheint es den Werbeverantwortlichen leichter zu fallen, ein Arzneimittel nicht mehr als "neu» zu bezeichnen, wenn es länger als ein Jahr auf dem Schweizer Markt ist, denn in nur noch 1 Fall (Vorjahr: 2) musste eine Korrektur verlangt werden.

Superlative und Komparative werden zunehmend verwendet. Aber die Interpretation der Regeln erweist sich oft als schwierig, was sich in einer Verdreifachung von 11 auf 33 Klagen wegen unzulässiger Vergleiche und Superlative (12, 1999: 6) zeigt. Die Abklärung, ob ein Vergleich zulässig ist, erweist sich durch das notwendige, umfangreiche Literaturstudium oft als zeitaufwendig, da vergleichende Aussagen zur Wirksamkeit auf kontrollierten Studien mit einem direkten Vergleich beider Substanzen beruhen müssen.

Unverändert 3 Fälle betrafen das Gebot, bei Geschenken und Gastfreundschaft massvoll zu bleiben. Die Kennzeichnung einer Aussendung als «Wichtige Mitteilung" war nur noch in 2 Fällen (1999: 6) unkorrekt (sie ist nur zur Wahrung der Arzneimittelsicherheit gestattet).

Aus Stichproben ergab sich, dass etliche Firmen bei der Erfüllung der Pflicht zur Zustellung von Belegexemplaren ihres Promotionsmaterials die nötige Sorgfalt vermissen lassen. Weil sie keinen Kodexverstoss betrafen, wies schliesslich der Sekretär 25 (Vorjahr: 39) Beanstandungen als nicht stichhaltig zurück.

Die Promotion für Arzneimittel wird besser, wenn sie kritisch gewürdigt wird, vor allem von den Adressaten. Wiederum seien darum alle Ärzte und Apotheker aufgefordert, spontan an den Sekretär zu gelangen, wenn ihnen eine Anzeige, eine Aussendung oder sonst eine Promotionsmassnahme aus ethischer oder wissenschaftlicher Sicht missfällt. Bei Bedarf kann der Pharma-Fachwerbungs-Kodex auf deutsch, französisch oder englisch auf der SGCIInternetseite (www.sgci.ch) abgerufen werden. 